\title{
Trace amounts of sporadically reappearing HCV RNA can cause infection
}

\author{
Naga Suresh Veerapu, ${ }^{1}$ Su-Hyung Park, ${ }^{1}$ Damien C. Tully, ${ }^{2}$ Todd M. Allen, ${ }^{2}$ and Barbara Rehermann ${ }^{1}$ \\ IImmunology Section, Liver Diseases Branch, National Institute of Diabetes and Digestive and Kidney Diseases (NIDDK), NIH, Department of Health and Human Services (DHHS), Bethesda, Maryland, USA. \\ ${ }^{2}$ Ragon Institute of MGH, MIT, and Harvard, Boston, Massachusetts, USA.
}

\begin{abstract}
Successful hepatitis C virus (HCV) treatment is defined as the absence of viremia 6 months after therapy cessation. We previously reported that trace amounts of HCV RNA, below the sensitivity of the standard clinical assay, can reappear sporadically in treatment responders. Here, we assessed the infectivity of this RNA and infused 3 chimpanzees sequentially at 9-week intervals with plasma or PBMCs from patients who tested positive for trace amounts of HCV RNA more than 6 months after completing pegylated IFN- $\alpha$ /ribavirin therapy. A fourth chimpanzee received HCV RNA-negative plasma and PBMCs from healthy blood donors. The 3 experimental chimpanzees, but not the control chimpanzee, generated HCV-specific T cell responses against nonstructural and structural HCV sequences 6-10 weeks after the first infusion of patient plasma and during subsequent infusions. In 1 chimpanzee, T cell responses declined, and this animal developed high-level viremia at week 27. Deep sequencing of HCV demonstrated transmission of a minor HCV variant from the first infusion donor that persisted in the chimpanzee for more than 6 months despite undetectable systemic viremia. Collectively, these results demonstrate that trace amounts of HCV RNA, which appear sporadically in successfully treated patients, can be infectious; furthermore, transmission can be masked in the recipient by an extended eclipse phase prior to establishing high-level viremia.
\end{abstract}

\section{Introduction}

At least 170 million people worldwide are persistently infected with hepatitis $\mathrm{C}$ virus (HCV), a leading cause of chronic inflammatory liver disease, cirrhosis, and cancer. The vast majority of patients who have been treated for chronic HCV infection received IFN-based treatment regimens. Pegylated interferon (PegIFN) in combination with ribavirin (RBV) has been the standard of care until the recent addition of direct-acting antivirals (1). A sustained virologic response (SVR) is defined as undetectable HCV RNA 6 months after the cessation of treatment. SVRs are considered cured because a virological relapse is exceedingly rare, and the risk of developing liver fibrosis and hepatocellular carcinoma decreases (2).

Considering the clinical experience of a long-term cure, it appears paradoxical that trace amounts of HCV RNA are sporadically detectable in the circulation (3) and in liver biopsies $(2,4-7)$ of some patients who experienced an SVR. Consistent with this, we recently reported that trace amounts of HCV RNA of pretreatment sequences, below the detection limit of the standard clinical assay at the NIH, reappeared sporadically in the blood of 15 of 98 (15\%) patients in the first 8 years after an SVR. The sporadic reappearance of HCV RNA was sufficient to recall HCV-specific T cell responses and did not result in high-level viremia (8).

At present, it is not clear whether this RNA represents replication-competent $\mathrm{HCV}$, whether it is associated with intact virions, and whether it can transmit infection. These questions are

Authorship note: Naga Suresh Veerapu and Su-Hyung Park contributed equally to this work.

Conflict of interest: The authors have declared that no conflict of interest exists Submitted: September 6, 2013; Accepted: May 29, 2014.

Reference information: J Clin Invest. 2014;124(8):3469-3478. doi:10.1172/JCI73104. of interest not only from epidemiological and infectious disease standpoints, but also from a virological standpoint. Based on our current virological knowledge, HCV should not be able to achieve low-level persistence over extended periods of time, because it is an RNA virus with a short 40-minute plasma half-life (9) and without the ability to integrate into the host genome. We therefore asked whether cryopreserved plasma and PBMCs from patients with an SVR to IFN-based therapy, in whom we had previously described sporadic recurrence of trace amounts of HCV, transmit HCV infection to chimpanzees and establish persistent infection. The results demonstrate that (a) such plasma can be infectious and establish high-level viremia and chronic hepatitis in the recipient, (b) the course of viremia in the recipient can differ from the typical course of acute hepatitis, in that HCV persists for more than 6 months in the absence of viremia prior to establishing highlevel systemic viremia, and (c) T cell responses correlate with temporary control of the low-level HCV infection.

\section{Results}

Trace amounts of HCV RNA that sporadically reappear in patients after successful antiviral therapy can transmit HCV infection. Three HCV-naive chimpanzees, A3A013, A3A015, and A3A017, were intravenously infused at 9-week intervals with human plasma or PBMCs (Table 1 and Supplemental Figure 1; supplemental material available online with this article; doi:10.1172/JCI73104DS1). These samples were derived from previously described anti-HCV-positive patients who had experienced an SVR to IFN-based therapy by qualitative COBAS Amplicor HCV Test 2.0 (the standard clinical test used at the NIH) but sporadically tested positive for trace amounts of HCV in plasma or PBMCs using a nested RT-PCR specific for the $5^{\prime}$ UTR of the virus (Table 1 and ref. 8). A fourth HCV-naive chimpan- 
Table 1. Characteristics of infused plasma and PBMCs

\begin{tabular}{|c|c|c|c|c|c|c|c|c|}
\hline \multirow{2}{*}{$\begin{array}{l}\text { Infused } \\
\text { chimpanzee }\end{array}$} & \multirow{2}{*}{$\begin{array}{l}\text { Infusion time } \\
\text { point (wk) }\end{array}$} & \multicolumn{3}{|c|}{ Infused biospecimen } & \multicolumn{4}{|c|}{ Source patient } \\
\hline & & Nature & $\begin{array}{l}\text { Plasma volume } e^{A} \\
\text { no. of cells }\end{array}$ & Source $^{B}$ & $\begin{array}{c}\text { HCV } \\
\text { genotype }\end{array}$ & $\begin{array}{l}\text { Time after therapy } \\
\text { cessation (yr) }\end{array}$ & $\begin{array}{l}\text { Therapy } \\
\text { regimen }\end{array}$ & $\begin{array}{l}\text { Therapy } \\
\text { duration }\end{array}$ \\
\hline \multirow[t]{3}{*}{ A3A025 } & 0 & Plasma & $22 \mathrm{ml}$ & Blood donor 1 & NA & NA & NA & NA \\
\hline & 18 & Plasma & $30.0 \mathrm{ml}$ & Blood donor 3 & NA & NA & NA & NA \\
\hline & 27 & PBMC & $35 \times 10^{6}$ & Blood donor 4 & NA & NA & NA & NA \\
\hline АЗАО13 & 0 & Plasma & $18.0 \mathrm{ml}(8 \mathrm{ml})$ & Patient 31 & $1 \mathrm{~b}$ & 0.7 & PegIFN/RBV & 48 \\
\hline & 18 & Plasma & $18.0 \mathrm{ml}(4 \mathrm{ml})$ & Patient 43 & $1 \mathrm{a}$ & 3.6 & PegIFN/RBV & 48 \\
\hline & 27 & PBMC & $35 \times 10^{6}$ & Patient 24 & $2 b$ & 0.9 & PegIFN/RBV & 24 \\
\hline \multirow[t]{4}{*}{ A3A015 } & 0 & Plasma & $24.0 \mathrm{ml}(6 \mathrm{ml})$ & Patient 67 & 2 & 2.4 & PegIFN/RBV & 24 \\
\hline & 9 & Plasma & $14.6 \mathrm{ml}(0.5 \mathrm{ml})$ & Patient 13 & 1 & 6.3 & IFN/RBV & 48 \\
\hline & 18 & Plasma & $20.0 \mathrm{ml}(6 \mathrm{ml})$ & Patient 40 & $1 \mathrm{~b}$ & 4.2 & PegIFN/RBV & 48 \\
\hline & 27 & PBMC & $35 \times 10^{6}$ & Patient 08 & $1 \mathrm{~b}$ & 2.6 & PegIFN/RBV & 48 \\
\hline & 27 & PBMC & $35 \times 10^{6}$ & Patient 13 & 1 & 6.3 & IFN/RBV & 48 \\
\hline
\end{tabular}

${ }^{A}$ Each plasma infusion consisted of plasma from a time point at which the patient tested positive by nested RT-PCR for trace amounts of HCV RNA below the detection limit of the qualitative COBAS Amplicor HCV Test 2.0 and the closest available time points at which the patient tested negative for HCV RNA. The first number indicates the total amount of infused plasma, and the number in parentheses indicates the volume from the HCV RNA-positive time point. ${ }^{B}$ Source patients are identified by the number used in a previous publication (8). NA, not applicable.

zee, A3A025, served as a control and was infused at the same time intervals with HCV RNA-negative plasma and PBMCs from blood donors without any history of HCV infection.

As shown in Figure 1, the control chimpanzee (A3A025) and 2 of the 3 experimental chimpanzees (A3A015 and A3A017) remained HCV RNA negative in the blood and liver throughout the entire study period, as determined by nested RT-PCR. Consistent with this finding, they did not display any significant increase in the intrahepatic expression levels of innate and adaptive immune response genes, such as OAS1 (Figure 1), IFIT1, MX1, CXCL1O, CXCL11, CD8B, and IFNG (data not shown). Alanine aminotransferase (ALT) activity, a marker of liver injury, remained within normal range throughout the study (Figure 1).

In contrast, the third experimental chimpanzee, A3A013, tested positive for trace amounts of HCVRNA by nested RT-PCRin theliver atweeks 9 and 20 (Figure2A), and intrahepatic expression of the IFNstimulated genes (ISGs) OAS1, IFIT1, MX1, CXCL10, and CXCL11 was increased at multiple time points (Figure 2). Systemic viremia $\left(2 \times 10^{6} \mathrm{HCV}\right.$ RNA copies/ml) and increased ALT levels (96 U/l) developed at week 27, the day of the fourth infusion (Figure 2A). Viral titers remained between $10^{4}$ and $10^{6} \mathrm{HCV}$ RNA for at least 6 months, indicating chronic HCV infection.

The transmitted HCV remains undetectable in the circulation for 27 weeks prior to causing high-level viremia and chronic infection. To determine the source of the high-level viremia in chimpanzee A3A013, we determined the genotype of the infecting virus from a week-20 liver biopsy and a week-29 serum sample from that chimpanzee and compared it with the HCV genotype in the source patients whose plasma and PBMC samples were infused. Based on the sequence of a 1.4-kb HCV 5'-UTR-core-E1 fragment, the infecting virus was genotype $1 \mathrm{~b}$. This excluded the second plasma infusion and the PBMC infusion as potential sources of infection, because these were derived from genotype 5 - and $2 b$-infected patients, respectively (Table 1). Phylogenetic analysis of the HCV sequences from chimpanzee A3A013 at weeks 20 and 29 and the HCV sequences of the remaining 2 source patients revealed that the infecting virus was most closely related to and thus likely transmitted by the first infusion sample (Figure 3, A and B). This was consistent with the detection of HCV RNA in the week-9 liver biopsy, which was obtained after the first and prior to the second plasma infusion (Figure 2A). The extended persistence of this variant in the liver in the absence of systemic viremia was also evidenced by the close sequence homology between the intrahepatic HCV sequence at week 20 and the HCV sequence in the circulation at week 29 (Figure 3).

Next, we performed quasispecies analysis by 454 pyrosequencing of the HCV E2-to-NS5B region. As shown in Figure $4 \mathrm{~A}$, we observed a greater $\mathrm{HCV}$ sequence diversity in the source patient (at the last time point during PegIFN/RBV therapy with an available serum sample and sufficient viremia for sequencing, i.e., less than 6 weeks prior to the first negative quantitative assay) than in the infected chimpanzee A3A013 (week 27 after infection, $P<0.0001)$. The dominant HCV E2-to-NS5B sequence in the chimpanzee at week 27 was most closely related to a minor quasispecies that represented $15.20 \%$ of the HCV sequences in the source patient (Figure 4B). Overall, 156 differences were identified among 7,800 nucleotides analyzed in the E2-to-NS5B region, translating into 38 amino acid differences between chimpanzee quasispecies Q1 and source patient quasispecies Q2. While the $\mathrm{HCV}$ nonstructural sequences were the most conserved between 
A

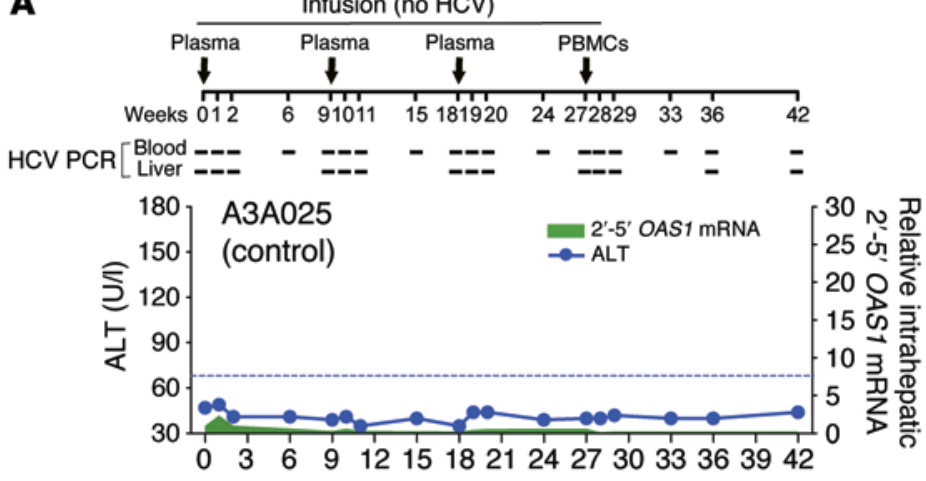

B Infusion of samples from patients with trace amounts of HCV
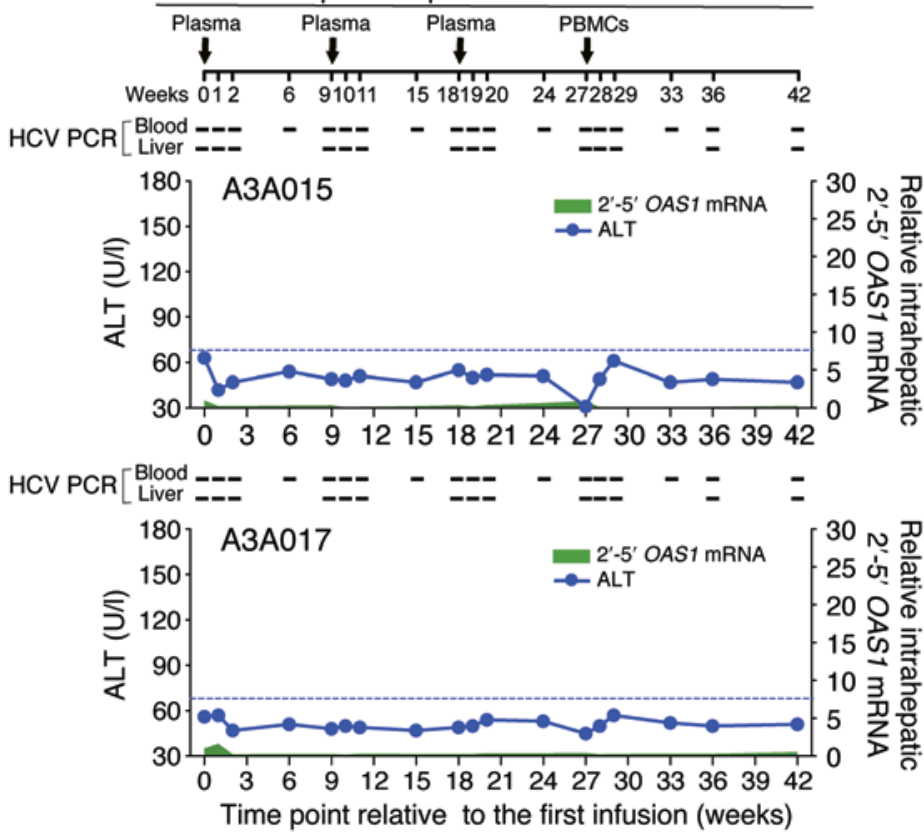

chimpanzee and patient, most of the sequence divergence was observed in the HCV HVR1 region located at the N terminus of E2 (Figure 4A). Consistent with the results for the entire E2-to-NS5B region, the chimpanzee HVR1 sequence was most closely related to a minor HVR1 sequence in the source patient's serum $(3.83 \%$ of all HVR1 sequences; Figure 4C), with only 9 amino acid differences between the 2 populations. This HVR1 variant was already detectable in the liver biopsy from chimpanzee A3A013 at week 20 after exposure (not shown), when there was no detectable systemic viremia, and it remained the dominant HVR1 variant when high titer viremia developed.

Taken together, these results demonstrate that trace amounts of HCV that sporadically reappear in the circulation of sustained virological responders to IFN-based therapy can be replication competent and transmit infection. Interestingly, the transmitted virus demonstrated the same phenotype of low-level persistence in the new host, remaining at low levels in the liver and undetectable in the circulation for 27 weeks.

The presence of HCV-specific T cells correlates with control of trace amounts of HCV. We performed a detailed analysis of humoral and cellular immune responses to understand (a) why only 1 of 3 ex-
Figure 1. Clinical follow-up of chimpanzees that remained aviremic after infusion of human plasma and PBMCs. (A) Chimpanzee A3A025 was intravenously infused at weeks 0,9 , and 18 with HCV RNA- negative plasma and at week 27 with HCV RNA-negative PBMCs from anti-HCV-negative blood donors. Each infused sample was derived from a different blood donor (Table 1). (B) Chimpanzees A3A015 and A3A017 were intravenously infused at weeks 0,9 , and 18 with plasma and at week 27 with PBMCs from anti-HCV-positive patients with trace amounts of HCV below the detection limit of the qualitative COBAS Amplicor HCV Test 2.0. Each infused sample was derived from a different patient (Table 1 and ref. 8). The presence of HCV RNA was assessed in blood and liver by qualitative RT-PCR. Intrahepatic expression of the ISG OAS1 was quantitated by real-time PCR. Intrahepatic IFIT1, MX1, CXCL10, CXCL11, CD8B, and IFNG mRNA levels were also quantitated by real-time PCR and did not increase over time (not shown). The 4 arrows in each graph indicate the 4 time points at which chimpanzees were infused with the biospecimens. The dotted blue line indicates the upper limit of normal of ALT levels.

perimental chimpanzees developed systemic viremia and (b) why there was an extended eclipse phase of 27 weeks prior to development of systemic viremia.

As shown in Figure 5A, HCV core-, NS3-, NS4-, and NS5-specific antibodies were transiently detectable in experimental chimpanzees A3A015, A3A017, and A3A013, but not in control chimpanzee A3A025, after some of the plasma infusions. These responses waned prior to each subsequent infusion (Figure 5A), suggesting that the antibodies were passively transferred with the infused plasma. Antibodies against HCV E2 proteins (of genotype 1a and $1 \mathrm{~b}$ sequences) were not detected at any time point during the study (Figure 5A).

Next, we examined T cell responses by assessing cytokine production by PBMCs in response to in vitro stimulation with overlapping HCV genotype 1a peptides. As shown in Figure 5B, the 3 experimental chimpanzees, but not the control chimpanzee, mounted HCV-specific T cell responses as early as week 6 after the first infusion (TNF- $\alpha$ in chimpanzee A3A017). To evaluate whether the use of genotype 1a peptides underestimated the immune response against inocula that contained HCV sequences other than genotype 1, we performed follow-up studies on chimpanzee A3A017, which was exposed to genotypes $1,2 \mathrm{~b}$, and 3 . Indeed, we observed stronger $\mathrm{T}$ cell responses at weeks 6,24 , and 33 , when NS3 peptides of genotype $2 b$ and 3 sequences were added to the respective pools of genotype 1a peptides. For example, there was no detectable PBMC response when genotype 1a peptides were used at week 6 after exposure (Figure $5 \mathrm{~B}$ ), while there was a response when genotype $2 \mathrm{~b}$ and 3 peptides were added (Figure 6A). The responses of all chimpanzees were predominantly mediated by $\mathrm{CD} 8^{+} \mathrm{T}$ cells and targeted both structural and nonstructural HCV sequences (Figure 6B). These results indicate that $\mathrm{HCV}$ replication occurred despite the absence of systemic viremia, because induction of $\mathrm{T}$ cell responses against nonstructural HCV antigens, which are not part of the viral particle, requires the translation of viral RNA in infected cells.

The HCV-specific $\mathrm{T}$ cell responses of chimpanzees A3A015 and $\mathrm{A} 3 \mathrm{~A} 017$ that remained aviremic throughout the study differed in 2 aspects from the HCV-specific T cell response of chimpanzee A3A013 that developed chronic, high-level HCV infection. 
A

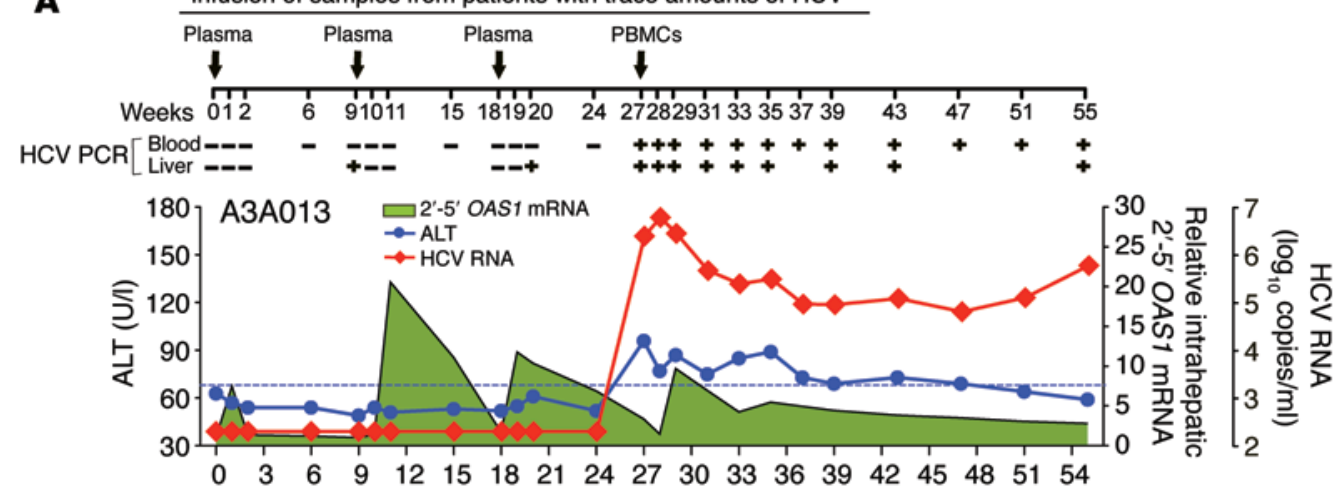

B
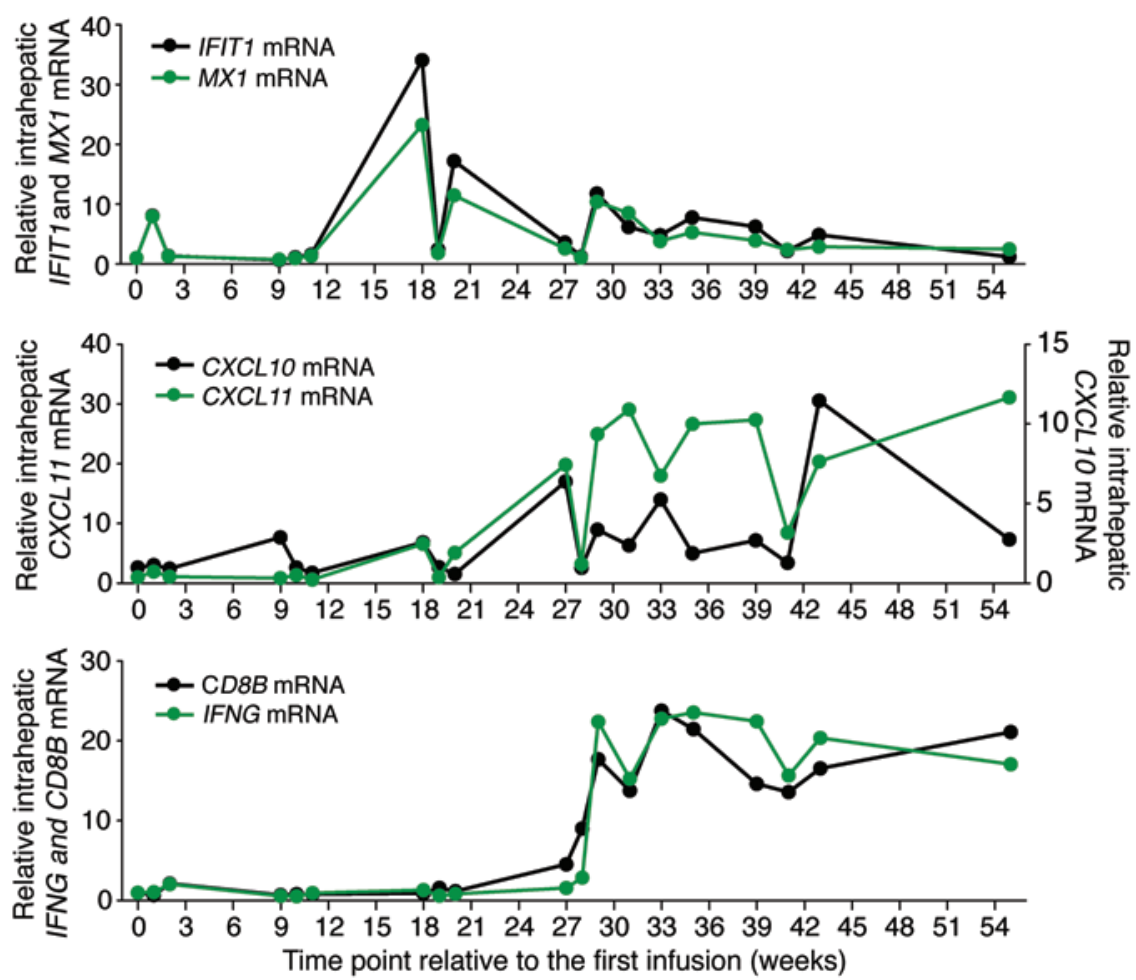

Figure 2. Clinical and virological follow-up of chimpanzee A3A013 that developed systemic viremia after infusion of human plasma and PBMCs. Chimpanzee A3A013 was intravenously infused at weeks 0,9 , and 18 with plasma and at week 27 with PBMCs from antiHCV-positive patients with trace amounts of HCV. Each infused sample was derived from a different patient (Table 1 and ref. 8). The 4 arrows indicate the 4 infusion time points. (A) The presence of HCV RNA was assessed in blood and liver by qualitative nested RT-PCR. - and + symbols indicate HCV RNA-negative and -positive samples, respectively. Serum HCV RNA levels, serum ALT levels, and intrahepatic OAS1 mRNA levels are indicated with a red line, a blue line, and a green area, respectively. The dotted blue line indicates the upper limit of normal of ALT levels. (B) Intrahepatic IFIT1, MX1, CXCL10, CXCL11, CD8B, and IFNG mRNA levels were determined by real-time PCR, normalized to endogenous references, and expressed as fold induction over week-0 values.
First, HCV-specific T cell responses from chimpanzees A3A015 and A3A017 were maintained throughout the study and were polyfunctional, as evidenced by secretion of multiple cytokines, whereas HCV-specific $\mathrm{T}$ cell responses from chimpanzee $\mathrm{A} 3 \mathrm{~A} 013$ were transient and produced fewer cytokines prior to the development of high-level viremia (Figure 5, B and C). Specifically, the absence of HCV-specific T cell responses at weeks 9 and 20 in chimpanzee A3A013 (Figure 5, B and C) coincided with the detection of HCV RNA in the liver biopsies at these time points (Figure $2 A)$. Furthermore, when $T$ cell responses disappeared for an extended time starting at week 18, chimpanzee A3A013 developed systemic high-level viremia with increased ALT values (Figure 2A) and seroconversion (Figure 5A). T cell responses were polyfunctional and stronger during this viremic phase than during the first 20 weeks but, again, were not maintained over time and did not prevent chronic $\mathrm{HCV}$ infection (Figure 5).

Taken together, these results suggest that HCV-specific T cells play a critical role in preventing the establishment of high-level systemic viremia after exposure to trace amounts of HCV.

\section{Discussion}

We previously described a window of time toward the end of $\mathrm{HCV}$ infection in which most of the HCV has been cleared, but traces of virus sporadically reappear in the circulation (8). These findings were made in a cohort of patients who sporadically tested positive for HCV RNA by nested RT-PCR within the first years after an SVR to IFN-based therapy (8). The results are consistent with reports showing residual virus in approximately $6 \%$ of SVRs by highly sensitive transcription-mediated amplification assays or nested RT-PCR $(2,10,11)$, even though a relapse with high levels of viremia is exceedingly rare (12-15).

Our study now provides proof-of-principle that these PCR signals do indeed reflect replication-competent infectious virus. Indeed, our study may have underestimated the transmission rate, because we had to re-use each chimpanzee for 4 sequential infusions. Thus, $\mathrm{HCV}$-specific $\mathrm{T}$ cell responses that were induced by the first infusion may have conferred protective immunity against subsequent infusions with human plasma and PBMCs with low levels of HCV. Consistent with this notion, chimpanzee 
A

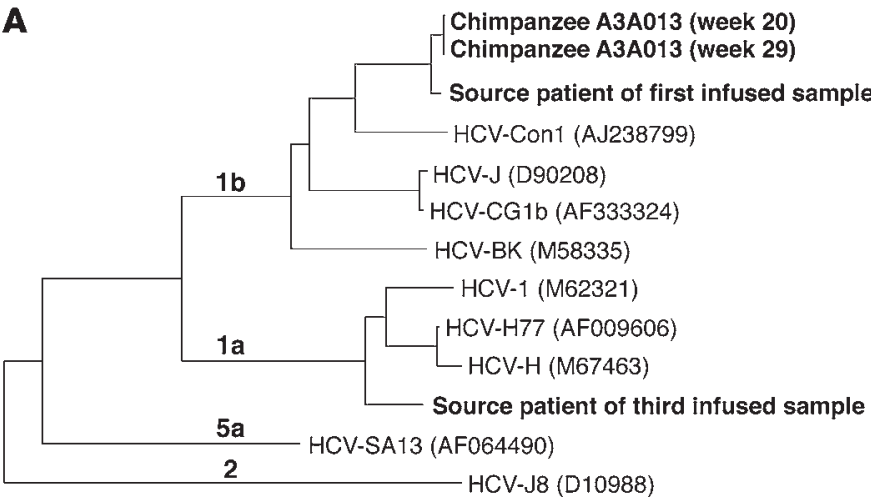

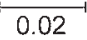

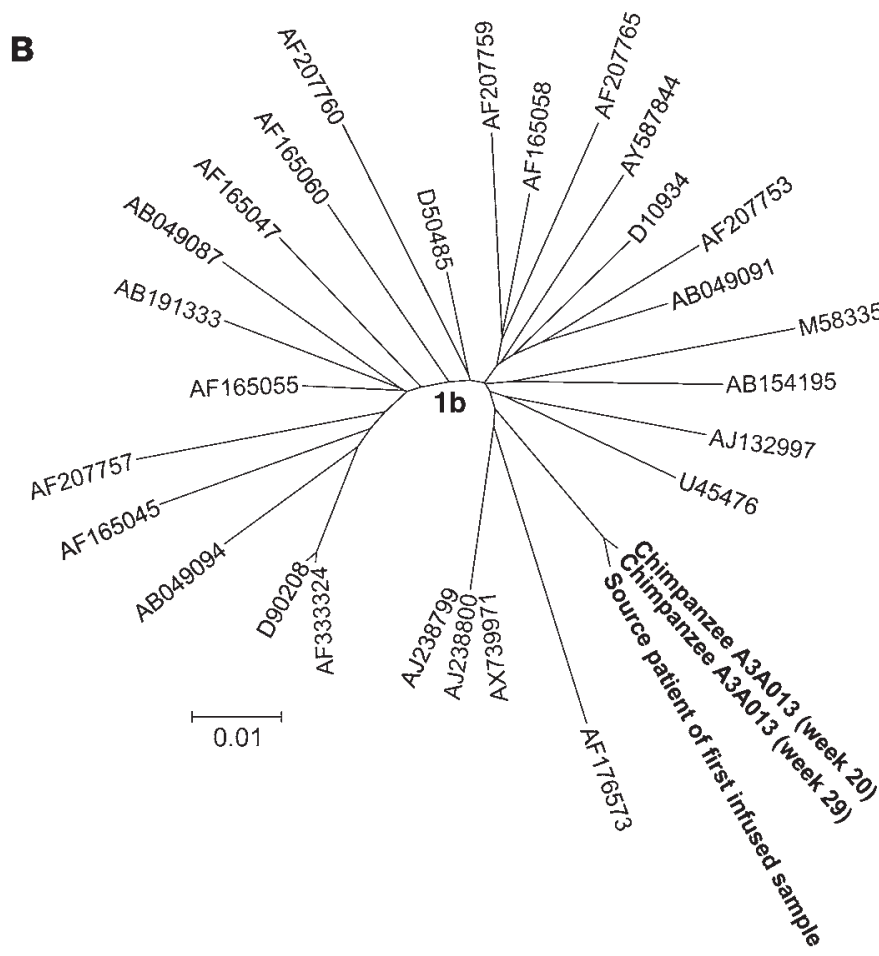

A3A013 developed systemic viremia only when HCV-specific $\mathrm{T}$ cell responses declined.

Although this study documents that sporadically detected HCV RNA can represent infectious virus, it should not be used to justify virological testing beyond 24 weeks after treatment in patients with an SVR. However, the observed delayed viremia after low-level HCV infection may warrant a longer follow-up of health care workers after HCV exposure. Consistent with the results of our study, a case report described 2 health care workers who developed viremia 5 months and 9 months after needlestick injuries, respectively (16). HCV-specific T cell responses were analyzed in 1 of these individuals and, as in our study, were found to decline just prior to the emergence of systemic viremia (16). Thus, the final testing for HCV RNA and HCV antibodies should occur more than 7 months after exposure, which is a longer follow-up than currently recommended by the Centers for Disease Control and Prevention (CDC) (17).
Figure 3. Comparison of HCV sequences of the sources of the inocula with the HCV sequence of infected chimpanzee A3A013. (A) Phylogenetic reconstruction of HCV sequences from donors and chimpanzee A3A013, which was infused with plasma samples from these donors. The sequence of a 1.2-kb HCV core-to-E1 fragment was amplified from chimpanzee A3A013's week-29 serum and week-20 liver biopsy and compared with the corresponding HCV sequences of the source patients of the first and third infusion, because they shared HCV genotype 1 with the infected chimpanzee. Reference sequences from common genotype $1 \mathrm{a}, 1 \mathrm{~b}, 2$, and $5 \mathrm{a}$ strains were included. The maximum likelihood tree was rooted with the genotype 2 sequence. The scale bar corresponds to 0.02 substitutions per site. (B) Phylogenetic reconstruction of HCV core-to-E1 sequences from chimpanzee A3A013's week-29 serum and week-20 liver biopsy and the source patient's plasma compared with various HCV genotype $1 \mathrm{~b}$ reference sequences. The scale bar corresponds to 0.01 substitutions per site.

The delayed appearance of systemic viremia may be due to several factors. First, the fact that HCV persisted in the absence of systemic viremia in both the source patient (8) and the infused chimpanzee in this study suggests that the unusual course of infection is due to the specific viral isolate rather than to host factors. While we excluded IFN resistance as described in a Japanese population due to mutations in the $\mathrm{N}$-terminal NS5A region (18), it is possible that HCV persists in hepatocytes that become refractory to IFN signaling $(19,20)$. Notably, the source patient experienced a very slow second-phase virological response to IFN-based therapy (not shown). Second, the low viral titer in the injected plasma may have delayed viremia. Recently, Asabe et al. showed delayed systemic viremia in chimpanzees that had been inoculated with $10^{1}$ genomic equivalents (GE) of $\mathrm{HBV}$ per milliliter as compared with those inoculated with $10^{7}$ and $10^{4} \mathrm{HBV}$ GE per milliliter (21). As in our study, injection of the low-dose inoculum resulted in chronic infection. Finally, our results support the interesting hypothesis that HCV persists in a form that is refractory to eradication by IFN-based - and possibly even direct-acting antiviral - therapy, as recently proposed by Ralston et al. (22). Consistent with this notion, Bauhofer et al. demonstrated in an in vitro study that long-term exposure of differentiated quiescent hepatoma cells to IFN- $\alpha$ reduced $\mathrm{HCV}$ replication 1,000-fold, but did not eliminate $\mathrm{HCV}$, and that viral replication rebounded after IFN- $\alpha$ withdrawal (23).

A final interesting aspect of this study is the immune response that the infected chimpanzees mounted. The passively transferred antibodies likely did not contribute much to the control of $\mathrm{HCV}$ infection, because they were unable to eliminate the autologous virus in the source patients, and because their titer decreased rapidly after each infusion in the chimpanzees. While the presence of strain-specific neutralizing antibodies cannot be formally excluded, the current data point to a role of the cellular immune response in the control of low-level HCV infection. Specifically, the induction of $\mathrm{T}$ cell responses against nonstructural HCV antigens that are not part of the HCV particle suggests that translation of HCV RNA occurred in infected cells. This was consistent with the detection of intrahepatic HCV RNA and ISG induction in chimpanzee A3A013. Chimpanzees A3A015 and A3A017, which also mounted $\mathrm{T}$ cell responses, either may have rapidly controlled 
A

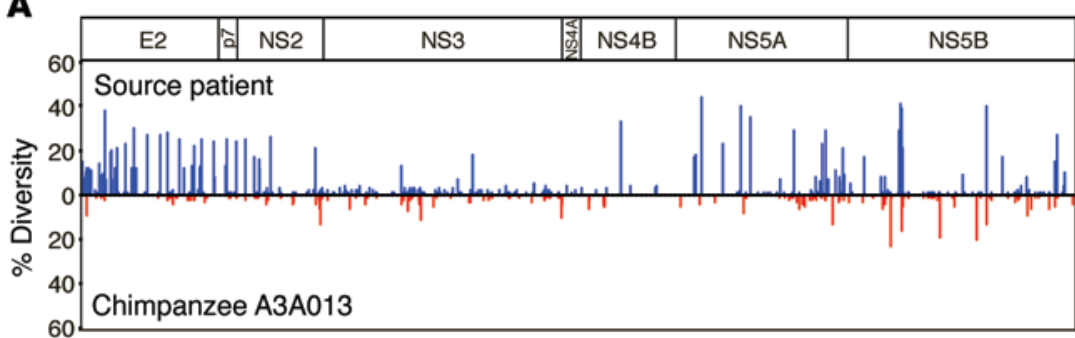

B

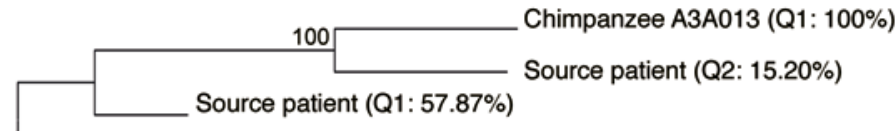

Source patient (Q3: 9.29\%)

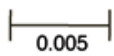

C

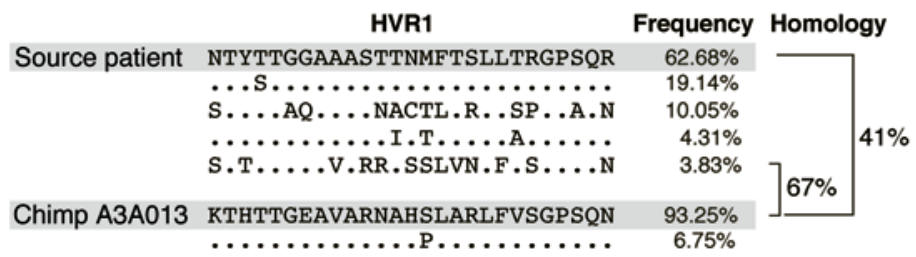

Figure 4. Transmission of a minor quasispecies with a unique HVR1 sequence. (A) Comparison of HCV amino acid sequence diversity in the source patient (at the last time point during PegIFN/RBV therapy at which the patient still had sufficient viremia to sequence $[11,360$ copies $/ \mathrm{ml}$ ], i.e., less than 6 weeks prior to the first negative quantitative assay) and in infected chimpanzee A3A013 (week 27). Plotted is the percentage of amino acid diversity at each position spanning E2 to NS5B with respect to the dominant amino acid found within each sample. (B) Comparison of the dominant HCV E2-toNS5B nucleotide sequence in chimpanzee A3A013 using the same biospecimens as in A. Percentages indicate the prevalence of the respective quasispecies among the detected sequences. The scale bar corresponds to 0.005 substitutions per site. (C) Comparison of the HCV HVR1 sequences in chimpanzee $\mathrm{A} 3 \mathrm{A013}$ and in the source patient using the same biospecimens as in $\mathbf{A}$. Sequences are shown relative to the dominant HVR variant in the source patient (top panel) or chimpanzee (bottom panel), with dots indicating conserved residues. The frequency of each HVR1 variant among the total number of HVR1 variants in the patient or chimpanzee sample is indicated. The dominant HVR sequence in the chimpanzee is most closely related to a minor quasispecies that represents $3.83 \%$ of the HVR sequences in the patient, with $67 \%$ amino acid sequence homology between the 2 . intrahepatic HCV RNA, or the biopsies may have sampled uninfected rather than infected hepatocytes.

The presence of $\mathrm{T}$ cell responses in the absence of viremia and seroconversion in the 3 experimental chimpanzees is reminiscent of the immune status of subgroups of injection drug users, health care workers, and family members of chronic HCV patients who are frequently exposed to low-level $\mathrm{HCV}$ but test negative for HCV RNA and antibodies (24-30). Of note, however, there was no substantial increase in the breadth or strength of the immune response after each exposure, which is consistent with attrition of memory $\mathrm{T}$ cell subpopulations after heterologous exposures (31, 32) and with the observation that the $T$ cell responses of the lowdose $\mathrm{HCV}$-exposed chimpanzees did not protect against highdose HCV challenge (33).

\section{Methods}

\section{Chimpanzees and experimental design}

Three naive chimpanzees, A3A013, A3A015, and A3A017, were intravenously infused at New Iberia Research Center (Lafayette, Louisiana, USA) with 15 to $31 \mathrm{ml}$ plasma from anti-HCV-positive patients who had tested negative for HCV RNA by the standard clinical assay used at the NIH (qualitative COBAS Amplicor HCV Test 2.0; Roche Molecular Diagnostics) but tested positive by nested RT-PCR specific for the HCV 5'-UTR (8). The infused chimpanzees remained negative for HCV RNA in the blood, as determined by RT-nested PCR at weeks 1,2 , and 6 after infusion and were subsequently infused at 9-week intervals with 2 plasma samples and 1 PBMC sample from 3 additional patients (Table 1). A control chimpanzee (A3A025) received, at the same time intervals, plasma and PBMCs from blood donors without any history of HCV. The 4 chimpanzees were not exposed to other chimpanzees with HCV infection at any time point during the study.
Starting with each inoculation, they were separated from each other until it was confirmed that they tested negative for HCV at the subsequent bleed date. Chimpanzee serum samples and snap-frozen liver biopsies were collected at the indicated time points (Figure 1, A and $\mathrm{B}$, and Figure $2 \mathrm{~A}$ ) and stored at $-80^{\circ} \mathrm{C}$. Acid citrate dextrose-anticoagulated blood tubes were shipped overnight to the NIH for isolation of PBMCs and plasma.

\section{Virological assays}

Qualitative RT-PCR. RNA was extracted from $1 \mathrm{ml}$ whole blood and $0.2 \mathrm{ml}$ plasma using the Roche High Pure Viral Nucleic Acid Large Volume Kit (Roche Diagnostics) and the QIAamp Viral RNA Mini Kit (QIAGEN), respectively. RT and qualitative nested PCR (detection limit, <40 HCV copies/ml) were performed as previously described (8), except that Superscript III (Invitrogen) and a primer amount of 2.5 pmol were used in the RT reaction. Standard precautions were taken to avoid contamination (34). In particular, samples from HCV-negative donors were included in extraction and in reverse and amplification steps, and RNA extraction and RT were performed in a different building than were the nested PCRs.

Quantitation of HCV RNA. Real-time PCR assays (detection limit, 200 copies/ml) were performed as previously described (35) using RNA that was extracted from $150 \mu \mathrm{l}$ serum with the QIAamp Viral RNA Mini Kit.

HCV sequence analysis using PCR products. HCV RNA was extracted from serum or liver biopsy samples using the High Pure Viral Nucleic Acid Large Volume Kit or the Arcturus PicoPure RNA Isolation Kit (Life Technologies), respectively. To sequence (a) full-length HCV from serum of the source patient and chimpanzee A3A013 at 29 weeks after the first HCV exposure and (b) HCV core-to-E1 from serum of the donor of the third infusion and from a liver biopsy of chimpanzee A3A013 at 20 weeks after the first $\mathrm{HCV}$ exposure, a 5.2-kb region encoding 

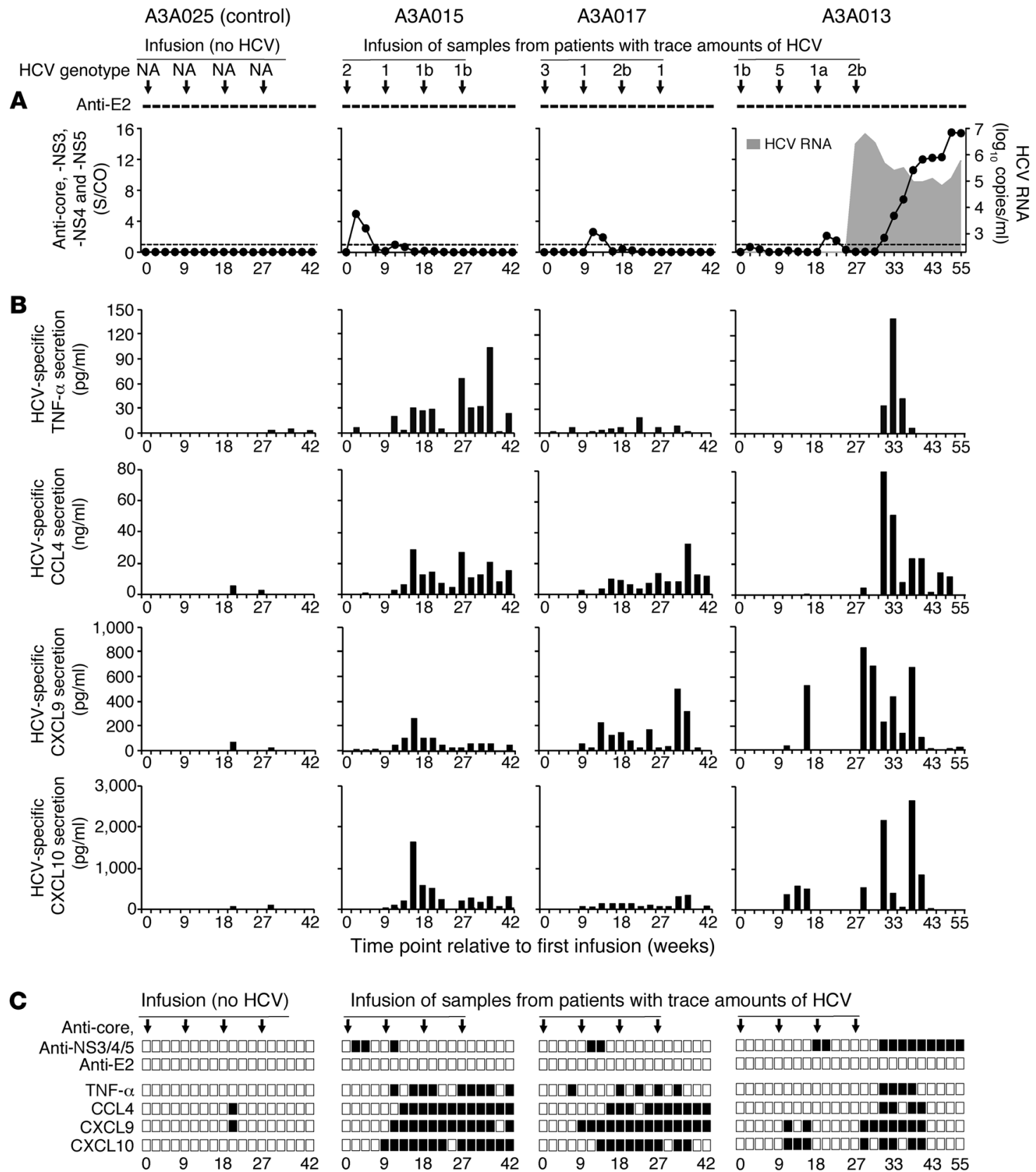

Infusion of samples from patients with trace amounts of HCV
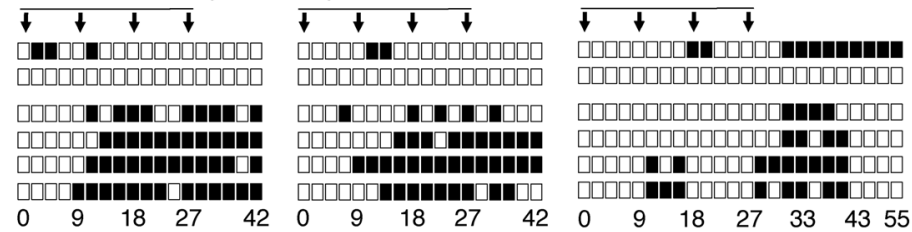

Time point relative to first infusion (weeks)

Figure 5. HCV-specific humoral and cellular immune responses of the infused chimpanzees. Chimpanzee A3A025 was intravenously infused at weeks 0, 9, and 18 with plasma and at week 27 with PBMCs from anti-HCV-negative and HCV RNA-negative blood donors. Chimpanzees A3A015, A3A017, and A3A013 were intravenously infused at weeks 0,9 , and 18 with plasma and at week 27 with PBMCs from anti-HCV-positive patients with trace amounts of HCV below the detection limit of the qualitative COBAS Amplicor HCV Test 2.0. Each infused sample was derived from a different patient (Table 1). The 4 small arrows in each graph indicate the 4 time points at which the chimpanzees were infused with human specimens. (A) Plasma samples were tested using Ortho ECi Vitros for HCV core, NS3, NS4, and NS5 antibodies. An anti-E2 EIA was performed as previously described (41). "-" indicates undetectable antibodies against HCV E2 of genotypes $1 \mathrm{a}$ and $1 \mathrm{~b}$. Shaded area indicates the HCV titer in the blood. (B) HCV-specific T cell responses were assessed by stimulating PBMCs with 18 pools of overlapping HCV peptides and quantitating TNF- $\alpha$, CCL4, CXCL9, and CXCL10 in the cell culture supernatant using a cytometric bead array assay. (C) Summary of HCV-specific antibody and T cell responses. Black squares indicate an immune response above the cutoff, and white squares indicate an absence of an immune response.

the HCV 5'-UTR to NS3/NS4A junction was reverse transcribed and amplified using nested PCR as described (36). For full-length HCV sequencing, an additional 4.5-kb region encoding HCV NS4 and NS5 was reverse transcribed using Superscript III (Invitrogen) with primer 5'-CTCAGGCCTATTGGCCTGGAG-3' and amplified by a nested
PCR using Platinum Taq DNA Polymerase High Fidelity (Invitrogen), first-round PCR primers 5'-GCATMTACAGGTTTGTGACTCC-3' and 5'-ATTGGCCTGGAGTGTTTAGC-3', and second-round PCR primers 5'-CATCGTGGGATCAAA TGTGG-3' and 5'-GCTGTGATATATGTCTCC-3'. Thermal cycling parameters for first- and second-round 
A
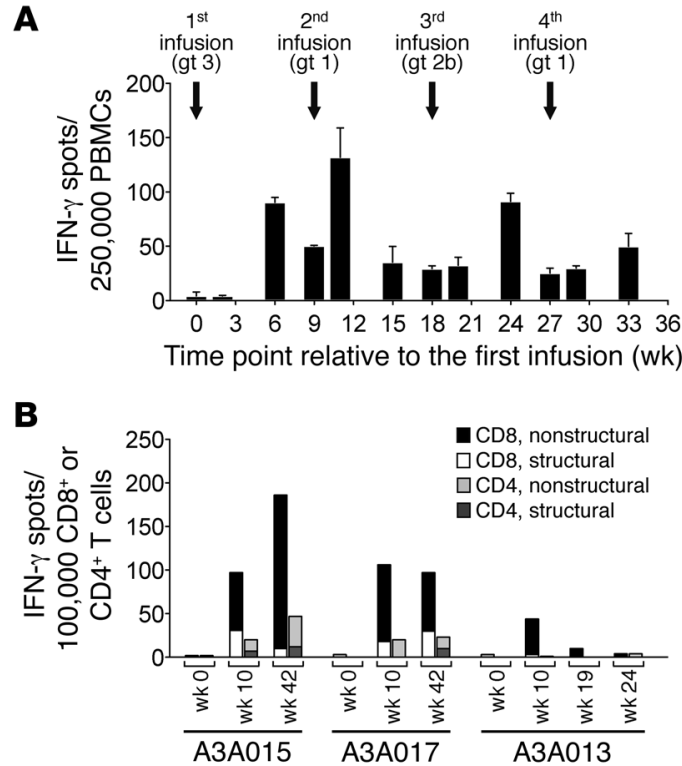

Figure 6. Specificity of $\mathrm{CD4}^{+}$and $\mathrm{CD8}^{+} \mathrm{T}$ cell responses. (A) PBMCs were obtained from chimpanzee A3A017 at the indicated time points and tested in an ex vivo IFN- $\gamma$ ELISPOT assay with 7 pools of HCV NS3 peptides. Each pool contained 14 overlapping 18-mer peptides of the HCV NS3 sequences of genotypes $1 \mathrm{a}, 2 \mathrm{~b}$, and 3 ( 42 peptides per pool). gt, genotype of the $\mathrm{HCV}$ inoculum. Mean $\pm \mathrm{SEM}$. (B) $\mathrm{CD} 4^{+}$and $\mathrm{CD} 8^{+} \mathrm{T}$ cells were isolated from PBMCs by magnetic cell separation and assessed for their specificity in IFN- $\gamma$ ELISPOT assays using 18 pools of overlapping HCV genotype $1 a$ peptides. Bars indicate the sum of the specific response to HCV structural and nonstructural antigens.

PCR were $95^{\circ} \mathrm{C}$ for 2 minutes, followed by 35 cycles of $94^{\circ} \mathrm{C}$ for $30 \mathrm{sec}-$ onds, $52^{\circ} \mathrm{C}$ for 35 seconds, and $68^{\circ} \mathrm{C}$ for 5 minutes, with a final extension step at $68^{\circ} \mathrm{C}$ for 5 minutes. Purified PCR products were directly sequenced with the ABI Prism BigDye Terminator Cycle Sequencing Reaction kit (Applied Biosystems) using reverse primers (listed in Supplemental Table 1). Sequence contigs were assembled with CodonCode Aligner (version 3.7.1.1; CodonCode Corporation), analyzed, and aligned using CLC Workbench 4, version 4.5.1 (CLC bio, QIAGEN).

\section{$\mathrm{HCV}$ quasispecies analysis by 454 pyrosequencing}

RNA was isolated from $140 \mu \mathrm{l}$ serum using the QIAamp Viral RNA Mini Kit (QIAGEN). Four overlapping amplicons covering HCV E2 to NS5B were generated from RNA using the SuperScript III One-Step RT-PCR System with Platinum Taq DNA Polymerase High Fidelity (Invitrogen) according to the manufacturer's protocol (primers are listed in Supplemental Table 1). Thermal cycling parameters were as follows: $55^{\circ} \mathrm{C}$ for 30 minutes, $94^{\circ} \mathrm{C}$ for 2 minutes, followed by 40 cycles of $94^{\circ} \mathrm{C}$ for 15 seconds, $58^{\circ} \mathrm{C}$ for 30 seconds, and $68^{\circ} \mathrm{C}$ for 4 minutes, with a final extension step at $68^{\circ} \mathrm{C}$ for 10 minutes, and then held at $10^{\circ} \mathrm{C}$. Purified PCR products from each sample were pooled in equimolar ratios, fragmented using the transposon-mediated Nextera DNA Sample Prep Kit (Illumina) following the manufacturer's protocol, and purified with a DNA Clean and Concentrator Kit (Zymo Research). Adapters and barcodes were added by limitedcycle PCR (Nextera). Small fragments were removed following Roche's recommended size selection protocol and quantified using a Promega Quantiflor-ST fluorometer. After quantification, barcoded samples were pooled at a final concentration of $10^{7}$ molecules/ $\mu$ lo create the library for sequencing on the GS Junior 454 Genome Sequencer (Roche Diagnostics). Emulsion PCR, breaking, and DNA sequencing were performed according to the manufacturer's protocols for Lib-L (Roche Diagnostics). Resulting sequence reads were then assembled into a de novo consensus using AV454 and VICUNA (37, 38). Alignments were generated using Mosaik (version 2.1.73), and reads were cleaned of carry-forward and incomplete extension (CAFIE) and homopolymer/frameshift errors using RC454. After cleaning, reads were passed to V-Phaser for variant calling. Briefly, V-Phaser uses phase and quality filtering with a probability model that recalibrates quality scores for individual bases to iteratively refine probabilities and to define the threshold required to statistically define a true variant from a sequencing artifact (39). Analyses were further subjected to manual inspection to identify and discard any sequencing artifacts. Global haplotype reconstruction was performed with PredictHaplo, version 5, using the default parameter settings. All consensus genome assemblies and annotations generated as part of this study were submitted to NCBI's GenBank database (KJ679443 and KJ679444).

\section{Phylogenetic analysis}

A phylogenetic tree was constructed using the neighbor-joining method from the Kimura 2-parameter distance matrix, and bootstrap values were determined from 1,000 bootstrap resamplings using MEGA software, version 5.05 (Center for Evolutionary Medicine and Informatics). Genotype 1a (HCV-1 [M62321], H77 [AF009606], HCV-H [M67463]), genotype 1b (BK [M58335], Con1 [AJ238799], CG1b [AF333324], HCV-J [D90208]), genotype 2 (HCV-J8 [D10988]), genotype 5A (HCV-SA13 [AF064490]) reference sequences (Figure $3 \mathrm{~A}$ ), and 27 genotype $1 \mathrm{~b}$ reference sequences that were arbitrarily chosen from Los Alamos HCV Sequence Data (http://hcv.lanl.gov) (Figure 3B) were included, respectively.

\section{Intrahepatic gene expression}

Snap-frozen liver biopsies were homogenized with a 0.5 -ml pestle and tube (Kimble Chase) in $300 \mu \mathrm{l}$ extraction buffer, and RNA was isolated using the PicoPure RNA Isolation Kit according to the manufacturer's instructions. Complementary DNA was synthesized using the MonsterScript 1st-Strand cDNA Synthesis Kit (Epicentre Biotechnologies). TaqMan Gene Expression Assays (Applied Biosystems) were performed in duplicate to determine OAS1, IFIT1, MX1, CXCL10, CXCL11, CD8B, and IFNG mRNA levels. The amount of specific mRNA was calculated using comparative cycle threshold values and standard curves (40), normalized to GAPDH and $\beta 7$ internal controls, and expressed as fold induction compared with week-0 data.

\section{HCV-specific T cell responses}

Freshly isolated PBMCs $\left(2 \times 10^{5} /\right.$ well $)$ were stimulated in RPMI1640 containing 5\% FBS, $100 \mathrm{IU} / \mathrm{ml}$ penicillin, $100 \mu \mathrm{g} / \mathrm{ml}$ streptomycin, and $2 \mathrm{mM} \mathrm{L-glutamine} \mathrm{(Mediatech),} \mathrm{with} 18$ pools of overlapping 15-mer peptides of the $\mathrm{HCV}$ genotype 1a (H77) sequence $(1 \mu \mathrm{g} / \mathrm{ml}$ per peptide, 600 peptides total), DMSO, or $25 \mu \mathrm{g} / \mathrm{ml}$ phytohemagglutinin (PHA-M) in 96-well round-bottom plates. Culture supernatants were harvested after 42 hours for quantitation of TNF- $\alpha$, CCL4, CXCL9, and CXCL10 using cytometric bead arrays (BD Biosciences) according to the manufacturer's protocol.

For IFN- $\gamma$ ELISPOT assays, $\mathrm{CD} 4^{+}$and $\mathrm{CD} 8^{+} \mathrm{T}$ cells were purified to greater than $95 \%$ from PBMCs by positive selection with magnetic beads (Miltenyi Biotec). ELISPOT assays were performed with $10^{5}$ 
$\mathrm{CD}^{+}$or $\mathrm{CD}^{+} \mathrm{T}$ cells per well in the presence of $2 \times 10^{5}$ irradiated (30 Gy) autologous CD4-CD8 ${ }^{-}$cells and 18 pools of overlapping HCV genotype 1a peptides $(1 \mu \mathrm{g} / \mathrm{ml}$ per each peptide, 600 peptides total), DMSO, or $1 \mu \mathrm{g} / \mathrm{ml}$ phytohemagglutinin (PHA-M) in RPMI1640 containing $5 \%$ FBS, penicillin, and glutamine, as described (8). In other IFN- $\gamma$ ELISPOT assays, $2.5 \times 10^{5}$ PBMCs were stimulated with 7 pools of HCV NS3 peptides. Each pool contained 14 overlapping 18-mer peptides of the respective genotype $1,2 b$, and 3 sequence ( 42 peptides per pool). After a 24-hour culture, plates were processed as described (8). Spots were counted using an AID ELISPOT Reader (Autoimmun Diagnostika), and the HCV-specific response was calculated by subtracting the mean number of spots in the negative control wells from the mean number of spots in the experimental wells.

\section{HCV-specific antibody responses}

Plasma samples were tested for HCV core, NS3, NS4, and NS5 antibodies using the Ortho ECi Vitros system (Ortho-Diagnostics). In addition, an anti-E2 enzyme immunoassay (EIA) was performed as previously described using genotype 1a and genotype 1b HCV E2 (41). An anti-E2 antibody response was considered positive if it was more than 2 times higher than the response at week 0 .

\section{Statistics}

Comparison of the sequence diversity within the source patient and chimpanzee A3A013 was performed using the Mann-Whitney $U$ test. A $P$ value of less than 0.05 was considered statistically significant.

\section{Study approval}

Chimpanzees were studied under protocols approved by the IACUC of the University of Louisiana at Lafayette (Lafayette, Louisiana, USA). Patients were studied under a protocol approved by the NIDDK/NIAMS institutional review board and provided written informed consent.

\section{Acknowledgments}

We thank T.J. Rowell, J. Fontenot, and the staff at New Iberia Research Center for the care of the chimpanzees and technical support; T. Jake Liang, Marc Ghany, and Theo Heller at the Liver Diseases Branch, NIDDK, NIH for patient samples and clinical information; and Colin Ogilvie and Karen Power at the Ragon Institute for technical support with 454 pyrosequencing. This work was supported by the intramural research program of the NIDDK, NIH (to B. Rehermann) and NIAID grant U19AI082630 (to T.M. Allen).

Address correspondence to: Barbara Rehermann, Immunology Section, Liver Diseases Branch, NIDDK, National Institutes of Health, 10 Center Drive, Bldg. 10, Room 9B16C, Bethesda, Maryland 20892, USA. Phone: 301.402.7144; E-mail: Rehermann@nih.gov.

Naga Suresh Veerapu's present address is: Department of Life Sciences, Shiv Nadar University, Village Chitera, District Gautam Budh Nagar, Uttar Pradesh, India.
1. Liang TJ, Ghany MG. Current and future therapies for hepatitis $\mathrm{C}$ virus infection. $\mathrm{NEngl} \mathrm{JMed}$. 2013;368(20):1907-1917.

2. Maylin S, et al. Eradication of hepatitis $C$ virus in patients successfully treated for chronic hepatitis C. Gastroenterology. 2008;135(3):821-829.

3. Welker MW, Zeuzem S. Occult hepatitis C: how convincing are the current data? Hepatology. 2009;49(2):665-675.

4. Carreno V, Pardo M, Lopez-Alcorocho JM, Rodriguez-Inigo E, Bartolome J, Castillo I. Detection of hepatitis C virus (HCV) RNA in the liver of healthy, anti-HCV antibody-positive, serum HCV RNA-negative patients with normal alanine aminotransferase levels. J Infect Dis. 2006;194(1):53-60.

5. Castillo I, Rodriguez-Inigo E, Lopez-Alcorocho JM, Pardo M, Bartolome J, Carreno V. Hepatitis C virus replicates in the liver of patients who have a sustained response to antiviral treatment. Clin Infect Dis. 2006;43(10):1277-1283.

6. Radkowski M, et al. Persistence of hepatitis C virus in patients successfully treated for chronic hepatitis C. Hepatology. 2005;41(1):106-114.

7. McHutchison JG, et al. Hepatic HCV RNA before and after treatment with interferon alone or combined with ribavirin. Hepatology. 2002;35(3):688-693.

8. Veerapu NS, Raghuraman S, Liang TJ, Heller T, Rehermann B. Sporadic reappearance of minute amounts of hepatitis $C$ virus RNA after successful therapy stimulates cellular immune responses. Gastroenterology. 2011;140(2):676-685.e1.

9. Guedj J, et al. Modeling shows that the NS5A inhibitor daclatasvir has two modes of action and yields a shorter estimate of the hepatitis C virus half-life. Proc Natl Acad Sci U S A. 2013;110(10):3991-3996.

10. George SL, Bacon BR, Brunt EM, Mihindukulasuriya KL, Hoffmann J, Di Bisceglie AM. Clinical, virologic, histologic, and biochemical outcomes after successful HCV therapy: a 5 -year follow-up of 150 patients. Hepatology. 2009;49(3):729-738.

11. Morishima C, et al. Interpretation of positive transcription-mediated amplification test results from polymerase chain reaction-negative samples obtained after treatment of chronic hepatitis C. Hepatology. 2008;48(5):1412-1419.

12. Lee WM, Polson JE, Carney DS, Sahin B, Gale M, Gale M Jr. Reemergence of hepatitis $C$ virus after 8.5 years in a patient with hypogammaglobulinemia: evidence for an occult viral reservoir. J Infect Dis. 2005;192(6):1088-1092.

13. Lin A, Thadareddy A, Goldstein MJ, Lake-Bakaar G. Immune suppression leading to hepatitis C virus re-emergence after sustained virological response. J Med Virol. 2008;80(10):1720-1722.

14. Fujii $\mathrm{H}$, et al. Relapse of hepatitis $\mathrm{C}$ in a pegylatedinterferon-alpha- $2 \mathrm{~b}$ plus ribavirin-treated sustained virological responder. Hepatol Res. 2010;40(6):654-660.

15. Koh C, et al. Long-term outcome of chronic hepatitis $\mathrm{C}$ after sustained virological response to interferon-based therapy. Aliment Pharmacol Ther. 2013;37(9):887-894.

16. Gruener NH, et al. Late appearance of hepatitis C virus RNA after needlestick injury: necessity for a more intensive follow-up. Infect Control Hosp Epidemiol. 2009;30(3):299-300.
17. US Public Health Service. Updated US Public Health Service guidelines for the management of occupational exposures to HBV, HCV, and HIV and recommendations for postexposure prophylaxis. MMWR Recomm Rep. 2001;50(RR-11):1-52

18. Enomoto N, et al. Comparison of full-length sequences of interferon-sensitive and resistant hepatitis $C$ virus $1 b$. Sensitivity to interferon is conferred by amino acid substitutions in the NS5A region. JClin Invest. 1995;96(1):224-230.

19. Pulverer JE, et al. Temporal and spatial resolution of type I and III interferon responses in vivo. J Virol. 2010;84(17):8626-8638.

20. Makowska Z, Duong FH, Trincucci G, Tough DF, Heim MH. Interferon-beta and interferonlambda signaling is not affected by interferoninduced refractoriness to interferon-alpha in vivo. Hepatology. 2011;53(4):1154-1163.

21. Asabe $S$, et al. The size of the viral inoculum contributes to the outcome of hepatitis B virus infection. J Virol. 2009;83(19):9652-9662.

22. Ralston R, Jacobson I, Scull M. The conundrum of relapse in STAT-C therapy: does HCV play the Red Queen or Rip Van Winkle? Semin Liver Dis. 2011;31(4):410-419.

23. Bauhofer O, Ruggieri A, Schmid B, Schirmacher P, Bartenschlager R. Persistence of HCV in quiescent hepatic cells under conditions of an interferon-induced antiviral response. Gastroenterology. 2012;143(2):429-438.e8.

24. Mizukoshi E, et al. Hepatitis C virus (HCV)-specific immune responses of long-term injection drug users frequently exposed to HCV. J Infect Dis. 2008;198(2):203-212.

25. Freeman AJ, et al. Prevalence of production of 


\section{RESEARCH ARTICLE}

virus-specific interferon-gamma among seronegative hepatitis C-resistant subjects reporting injection drug use. JInfect Dis. 2004;190(6):1093-1097.

26. Thurairajah $\mathrm{PH}$, et al. Hepatitis $\mathrm{C}$ virus (HCV)-specific T cell responses in injection drug users with apparent resistance to HCV infection. J Infect Dis. 2008;198(12):1749-1755.

27. Zeremski M, et al. Hepatitis C virus-specific T-cell immune responses in seronegative injection drug users. JViral Hepat. 2009;16(1):10-20.

28. Heller T, et al. Occupational exposure to hepatitis $\mathrm{C}$ virus: early T-cell responses in the absence of seroconversion in a longitudinal cohort study. J Infect Dis. 2013;208(6):1020-1025.

29. Al-Sherbiny M, et al. Exposure to hepatitis C virus induces cellular immune responses without detectable viremia or seroconversion. Am J Trop Med Hyg. 2005;73(1):44-49.

30. Scognamiglio $P$, et al. Presence of effector $\mathrm{CD} 8+\mathrm{T}$ cells in hepatitis $\mathrm{C}$ virus-exposed healthy seronegative donors. J Immunol. 1999;162(11):6681-6689.

31. Welsh RM, Selin LK. Attrition of memory CD8 T cells. Nature. 2009;459(7247):E3-E4.

32. Bukh J, et al. Previously infected chimpanzees are not consistently protected against reinfection or persistent infection after reexposure to the identical hepatitis $C$ virus strain. J Virol. 2008;82(16):8183-8195.

33. Park SH, et al. Subinfectious hepatitis C virus exposures suppress $\mathrm{T}$ cell responses against subsequent acute infection. Nat Med. 2013;19(12):1638-1642.

34. Kwok S, Higuchi R. Avoiding false positives with PCR. Nature. 1989;339(6221):237-238.

35. Takeuchi T, et al. Real-time detection system for quantification of hepatitis C virus genome. Gastroenterology. 1999;116(3):636-642.

36. Liu Z, et al. Accurate representation of the hepatitis $C$ virus quasispecies in 5.2-kilobase ampli- cons. J Clin Microbiol. 2004;42(9):4223-4229.

37. Henn MR, et al. Whole genome deep sequencing of HIV-1 reveals the impact of early minor variants upon immune recognition during acute infection. PLoS Pathog. 2012;8(3):e1002529.

38. Yang $\mathrm{X}$, et al. De novo assembly of highly diverse viral populations. BMC Genomics. 2012;13:475.

39. Macalalad AR, et al. Highly sensitive and specific detection of rare variants in mixed viral populations from massively parallel sequence data. PLoS Comput Biol. 2012;8(3):e1002417.

40. Schmittgen TD, Livak KJ. Analyzing real-time PCR data by the comparative C(T) method. Nat Protoc. 2008;3(6):1101-1108.

41. Raghuraman S, Park H, Osburn WO, Winkelstein E, Edlin BR, Rehermann B. Spontaneous clearance of chronic hepatitis $C$ virus infection is associated with appearance of neutralizing antibodies and reversal of T-cell exhaustion. J Infect Dis. 2012;205(5):763-771. 